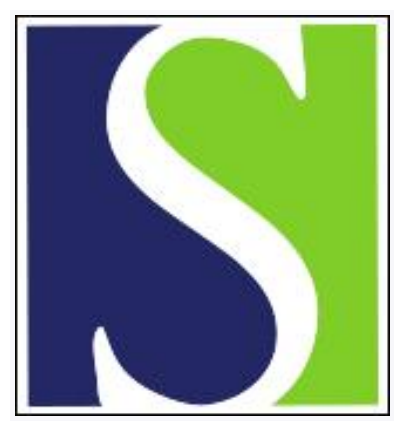

Scand J Work Environ Health 2009;35(3):233-240

https://doi.org/10.5271/sjweh.1317

Published online: 17 Apr 2009, Issue date: 00 May 2009

Lifetime occupational history and risk of endometriosis

by Marino JL, Holt VL, Chen C, Davis S

Affiliation: Discipline of Obstetrics and Gynaecology, University of Adelaide, Adelaide SA 5000, Australia. jennifer.marino@adelaide.edu.au

The following article refers to this text: 2016;42(1):52-60

Key terms: case-control study; endometriosis; health; occupational exposure; occupational history; risk; woman

This article in PubMed: www.ncbi.nlm.nih.gov/pubmed/19377833 


\title{
Lifetime occupational history and risk of endometriosis
}

\author{
by Jennifer L Marino, PhD, ${ }^{1,2,3}$ Victoria L Holt, PhD, ${ }^{1,2}$ Chu Chen, PhD, ${ }^{1,2}$ Scott Davis, PhD ${ }^{1,2}$
}

\begin{abstract}
Marino JL, Holt VL, Chen C, Davis S. Lifetime occupational history and risk of endometriosis. Scand J Work Environ Health 2009;35(3):233-240.

Objectives Endometriosis is the presence of functioning endometrial glands and stroma outside the uterine cavity, most often in the pelvic peritoneal cavity. Women with endometriosis commonly have dysmenorrhea, dyspareunia, pain, menorrhagia, and/or metrorrhagia. Disease complications can include adhesions, chronic pain, and infertility. In this exploratory case-control study, we investigated the relationship between lifetime occupational history and surgically confirmed endometriosis in a population-based sample.
\end{abstract}

Methods We conducted interviews with participants, all reproductive-aged female members of a large healthmaintenance organization who were first diagnosed with surgically confirmed endometriosis between April 1, 1996 and March 31, 2001. Interviews were also conducted with randomly selected controls, reproductive-aged female enrollees of the same organization from the same time period. Each reported job was coded using US Census Occupations and Industries codes, and classified into categories. We used unconditional logistic regression to compare having worked in a given job class with never having done so.

Results Our study found that an increased risk of endometriosis was associated with having worked as a flight attendant, service station attendant, or health worker, particularly as a nurse or health aide (flight attendant: odds ratio (OR) 9.80, 95\% CI 1.08-89.02; service station attendant: OR 5.77, 95\% CI 1.03-32.43; health worker: OR 1.49, 95\% CI 1.03-2.15). Income and education did not make a difference in the OR estimates for the occupations examined.

Conclusions This exploratory study suggested that there might be an associated risk of endometriosis for those women who have worked as a flight attendant, service station attendant, or health worker, particularly a nurse.

Key terms case-control study; health; occupational exposure; woman.

Endometriosis, which affects $5-10 \%$ of women of reproductive age in the United States, is the presence of functioning endometrial glands and stroma outside the uterine cavity, most often in the pelvic peritoneal cavity (1). It is a symptomatic disease that commonly features dysmenorrhea, dyspareunia, pain, menorrhagia, and/or metrorrhagia; complications can include adhesions, chronic pain, and infertility. Endometriosis is associated with an increased overall cancer risk (2), with particular elevation in risk of ovarian cancer (3-6).

Endometriosis has been studied as a potential outcome of occupational exposure to dioxins and included as a secondary outcome in general studies of reduced fertility among workers in certain industries and occupations. These studies have found an increased risk of endometriosis-associated infertility among workers exposed to formaldehyde (7), video display terminals (8), chemical dusts (8), or organic solvents $(7,8)$, and among workers in agricultural industries and occupations, in particular farm workers (9). To our knowledge there has been no examination to date of the full spectrum of occupational risk of endometriotic disease. In the United States, $72 \%$ of women age $25-64$ years work in the civilian labor force, and the majority of these work full-time all year (10). If occupational risks for endometriosis exist, such exposures will affect many women. This analysis explored the relationship between occupation and endometriosis in a large population-based case-control study.

1 Department of Epidemiology, School of Public Health and Community Medicine, University of Washington, Seattle, Washington, United States.

2 Division of Public Health Sciences, Fred Hutchinson Cancer Research Center, Seattle, Washington, United States.

3 Disciplines of Obstetrics and Gynaecology and Public Health, University of Adelaide, Adelaide, SA, Australia.

Correspondence to: Dr JL Marino, Discipline of Obstetrics and Gynaecology, University of Adelaide, Adelaide SA 5000, Australia. [E-mail: jennifer.marino@adelaide.edu.au] 


\section{Study population and methods}

\section{Study design}

As described elsewhere (11), we opened participation in our study to all female members of Group Health Cooperative, a large health-maintenance organization in Washington State, USA, age 18-49 years and first diagnosed with surgically confirmed endometriosis between April 1, 1996 and March 31, 2001 (N=467). The reference dates of potential cases were the month and year of the first Group Health Cooperative visit for symptoms ultimately leading to the diagnosis of endometriosis. Potential controls ( $\mathrm{N}=1016)$ were randomly selected from a list of women enrolled in Group Health Cooperative during the same time period, frequencymatched on a five-year age group, and assigned reference dates corresponding to the distribution of case reference dates.

A total of $341(73.0 \%)$ subjects and $742(73.0 \%)$ controls participated in the study. A face-to-face or telephone interview using a structured questionnaire collected information concerning exposures prior to the reference date, as well as demographics, prior medical conditions, reproductive history, and other characteristics. Participants' in- and out-patient medical records were reviewed and abstracted for type and severity of symptoms (eg, dysmenorrhea, menorrhagia), and endometriosis characteristics (eg, dimensions, presence of adhesions). Case definitions were further refined and participants whose disease did not meet the Holt-Weiss standards for definite or probable disease were excluded from this analysis (12). Briefly, under these criteria, ovarian endometriomas, pelvic endometriotic lesions over $5 \mathrm{~mm}$ deep, and pelvic endometriotic lesions with adhesions not attributable to other causes are classed as "definite disease", while other endometriotic implants with at least one major endometriosis symptom (eg, infertility, moderate to severe dysmenorrhea, moderate to severe dyspareunia, or moderate to severe pelvic pain) are classed as "probable disease". Therefore, cases without surgical evidence of disease and asymptomatic cases with superficial or ambiguous pelvic lesions $(\mathrm{N}=12)$ were excluded from the study. Additionally, three cases of extrapelvic scar endometriosis were excluded.

Subjects $(\mathrm{N}=13)$ and controls $(\mathrm{N}=14)$ who reported a previous surgically confirmed endometriosis diagnosis were excluded from this analysis as well. After all exclusions, 313 cases and 727 controls remained for this analysis. This study was approved by the institutional review boards of Fred Hutchinson Cancer Research Center and Group Health Cooperative. Each participant gave written informed consent to participate in the study and was compensated $\$ 20$ for her time.

\section{Exposure classification}

All participants were asked about each paid job they had held, part-time or full-time - for six months or longer - between 18 years of age and the reference date. Information collected included the job title, type of business or industry, average hours worked per week, and number of years worked at each job. Jobs were coded using the 1980 Census - Occupations and Industries codes (13, 14). These codes were grouped into 56 categories using the Schnitzer et al method (15). To improve statistical power, multiple Schnitzer et al categories were merged to form two broader classes: "wood- and paper-related jobs" and "health workers". The Schnitzer et al categories "forestry and logging workers", "sawmill workers", "paper workers" and "carpenters/woodworkers", which respectively had 7, 4, 3, and 6 participants, were combined into a new category "wood- and paperrelated jobs" $(\mathrm{N}=19)$. [Numbers do not sum because a respondent could work more than one job class during her history.] A new category, "health workers", consisted of the Schnitzer et al categories "dentists/dental technicians" ( $N=13$, all of whom were dental technicians), "physicians/surgeons" $(\mathrm{N}=3)$, and "nurses/health technicians" ( $\mathrm{N}=149)$. Within the "health worker" category, subcategories were created for (i) physicians/surgeons (job code 084), (ii) registered nurses/licensed practical nurses (job codes 095 and 207), (iii) radiological technician (job code 206), (iv) nursing/health aides (job codes 446 and 447) and (v) all other health workers. The Schnitzer et al category "aircraft operators" (occupation codes 226 and 465), was renamed "flight attendants" after examination of the individual jobs revealed that all of the respondents in this category listed their jobs as flight attendants (occupation code 465). These changes reflect the adaptation of the Schnitzer et al tool to an exclusively female population of workers, rather than the mixed-gender group originally used by Schnitzer et al, or the all-male population of the Canadian system from which the instrument emerged.

\section{Statistical analysis}

We used unconditional logistic regression to calculate odds ratios (OR) and $95 \%$ confidence intervals $(95 \%$ CI) for the association between endometriosis and occupation using STATA 8.2 (Stata Corp LP, College Station, TX, USA). Those who had ever worked in an occupation in a Schnitzer et al category were compared with all those who had never worked in that category. Using a low-stringency standard of $\alpha<0.15$, occupational groups that were significantly associated with endometriosis were examined more closely, and divided or further aggregated to obtain reasonably sized and meaningful categories for further analysis. 
Potential confounding factors considered in the creation of logistic regression models were race or ethnicity, household income, education, marital status, gravidity, parity, and use of alcohol, cigarettes, and oral contraceptives. Each potential confounder, treated categorically, was evaluated individually in a model containing each re-aggregated occupational group and the frequency-matching variables (age and reference year), to determine which, if any, changed the estimate of the association between the occupation and endometriosis by $10 \%$ or more. Those confounders were then included in a multivariate model to determine a final, conservatively adjusted estimate of association.

As women who seek treatment for infertility may have endometriosis discovered incidentally as part of the diagnostic process, rather than the result of symptomatic disease, we also considered separately cases who reported seeking care only for reasons other than infertility $(\mathrm{N}=260)$. Presentation of endometriosis was determined by the case participants' answers to the question, "on or before [the reference date], what was the reason you first visited a doctor or other health care practitioner that led to your recent diagnosis of endometriosis?" and their sexual intercourse histories. Those who listed infertility as one reason $(\mathrm{N}=36)$ or the only reason $(\mathrm{N}=17)$ for their initial visit were classified as having infertilityassociated endometriosis. Pre-menopausal women who reported 12 or more consecutive months of unprotected sexual intercourse with a male partner without a pregnancy immediately before their reference date, and who had no other reason for their initial visit, were also classed as having infertility-associated endometriosis.

\section{Results}

Demographic characteristics are shown in table 1. Cases and controls were similarly distributed in terms of age, race or ethnicity, marital status, education, income, and use of oral contraceptives. Cases had fewer pregnancies and fewer births than controls. Cases were more likely than controls to use tobacco or alcohol at the reference date.

Cases and controls were equally likely to have worked outside the home [305 (97.4\%) cases, 710 $(97.7 \%)$ controls, OR 1.01, 95\% confidence interval (95\% CI) $0.41-2.48$, after adjustment for reference age and reference year].

The distribution of Schnitzer et al's occupational categories among cases and controls is reported in table 2. Under the non-stringent criterion of $\alpha<=0.15$, the categories "biological scientists", "teachers, librarians", "aircraft operators", and "service station attendants" were significantly associated with endometriosis case status after adjustment for matching variables age and reference year. These categories were further examined in multivariate models, as were the categories that were reconstructed to add meaning and power (table 3). Potential confounders that made a $10 \%$ or greater difference in any "ever/never" risk estimate were alcohol use, oral contraceptive use, gravidity, and nulliparity. All "ever/never" models were thus adjusted for these variables as well as for age and reference year. In these multivariate models, health workers, service station attendants, and flight attendants were

Table 1. Sociodemographic and health characteristics of case and control participants.

\begin{tabular}{|c|c|c|c|c|}
\hline & \multicolumn{2}{|c|}{ Cases } & \multicolumn{2}{|c|}{ Controls } \\
\hline & $\mathrm{N}$ & $\%$ & $\mathrm{~N}$ & $\%$ \\
\hline \multicolumn{5}{|l|}{ Age } \\
\hline$<20$ years & 10 & 3.2 & 22 & 3.0 \\
\hline $20-29$ years & 44 & 14.1 & 94 & 12.9 \\
\hline $30-39$ years & 105 & 33.5 & 251 & 34.5 \\
\hline 40-49 years & 154 & 49.2 & 360 & 49.5 \\
\hline \multicolumn{5}{|l|}{ Race/ethnicity } \\
\hline Non-Hispanic Caucasian & 251 & 80.2 & 588 & 81.0 \\
\hline Non-Hispanic African-American & 11 & 3.5 & 38 & 5.2 \\
\hline Non-Hispanic Asian/Pacific Islander & 20 & 6.4 & 48 & 6.6 \\
\hline Non-Hispanic other & 9.0 & 2.9 & 30 & 4.1 \\
\hline Hispanic & 22 & 7.0 & 22 & 3.0 \\
\hline \multicolumn{5}{|l|}{ Highest educational level completed } \\
\hline$<12$ years & 11 & 3.5 & 23 & 3.2 \\
\hline 12 years & 56 & 17.9 & 136 & 18.7 \\
\hline Some college & 102 & 32.6 & 268 & 36.9 \\
\hline College graduate & 92 & 29.4 & 163 & 22.4 \\
\hline Post graduate & 52 & 16.6 & 137 & 18.8 \\
\hline \multicolumn{5}{|l|}{ Nulliparity } \\
\hline No births & 150 & 47.9 & 208 & 28.6 \\
\hline Any births & 163 & 52.1 & 519 & 71.4 \\
\hline \multicolumn{5}{|l|}{ Gravidity } \\
\hline 0 pregnancies & 118 & 37.7 & 154 & 21.2 \\
\hline 1 pregnancies & 45 & 14.4 & 121 & 16.6 \\
\hline 2 pregnancies & 73 & 23.3 & 185 & 25.5 \\
\hline 3 births or more & 77 & 24.6 & 267 & 36.7 \\
\hline \multicolumn{5}{|l|}{ Tobacco use } \\
\hline Never & 175 & 55.9 & 436 & 60.1 \\
\hline Former & 73 & 23.3 & 170 & 23.4 \\
\hline Current & 65 & 20.8 & 120 & 16.5 \\
\hline \multicolumn{5}{|l|}{ Alcohol use } \\
\hline Never & 92 & 29.4 & 258 & 35.5 \\
\hline Former & 62 & 19.8 & 162 & 22.3 \\
\hline Current & 159 & 50.8 & 307 & 42.2 \\
\hline \multicolumn{5}{|l|}{ Oral contraceptive use } \\
\hline Never & 35 & 11.2 & 117 & 16.1 \\
\hline Former & 237 & 75.7 & 518 & 71.3 \\
\hline Current & 41 & 13.1 & 92 & 12.7 \\
\hline Total & 313 & 100 & 727 & 100 \\
\hline
\end{tabular}


Table 2. Relationships between endometriosis case status and occupation for all Schnitzer et al occupational categories, all cases and cases seeking care for reasons other than infertility. ( $\mathrm{OR}=$ odds ratio, $95 \% \mathrm{Cl}=$ confidence interval)

\begin{tabular}{|c|c|c|c|c|c|c|c|}
\hline \multirow[t]{2}{*}{ Schnitzer et al occupational category a } & \multicolumn{2}{|c|}{ Cases } & \multicolumn{2}{|c|}{ Controls } & \multirow[t]{2}{*}{ OR } & \multirow[t]{2}{*}{$95 \% \mathrm{Cl}$} & \multirow[t]{2}{*}{ P-value } \\
\hline & $\mathrm{N}$ & $\%$ & $\mathrm{~N}$ & $\%$ & & & \\
\hline Managers, administrators & 92 & 29.4 & 222 & 30.5 & 0.96 & $0.72-1.29$ & 0.78 \\
\hline Engineers, architects, draughtsmen & 10 & 3.2 & 30 & 4.1 & 0.78 & $0.37-1.61$ & 0.49 \\
\hline Surveyors, geologists, prospectors & 0 & & 4 & 0.5 & . & .. & . \\
\hline Mathematical, physical and computer scientists & 24 & 7.7 & 52 & 7.1 & 1.10 & $0.66-1.83$ & 0.71 \\
\hline Biological scientists & 13 & 4.1 & 17 & 2.3 & 1.83 & $0.88-3.81$ & 0.11 \\
\hline Chemists, pharmacists, chemical engineers & 4 & 1.3 & 6 & 0.8 & 1.56 & $0.44-5.57$ & 0.49 \\
\hline Engineering, science technicians & 9 & 2.9 & 17 & 2.3 & 1.24 & $0.55-2.82$ & 0.60 \\
\hline Dentists, dental technicians & 6 & 1.9 & 7 & 1.0 & 2.02 & $0.67-6.06$ & 0.21 \\
\hline Physicians, surgeons & 2 & 0.6 & 1 & 0.1 & 4.68 & $0.42-51.85$ & 0.21 \\
\hline Nurses, health technicians & 52 & 16.6 & 101 & 13.9 & 1.25 & $0.87-1.80$ & 0.23 \\
\hline Teachers, librarians & 53 & 16.9 & 158 & 21.7 & 0.74 & $0.52-1.05$ & 0.09 \\
\hline Legal and social service workers & 27 & 8.6 & 66 & 9.1 & 0.96 & $0.60-1.53$ & 0.86 \\
\hline Entertainers, athletes & 14 & 4.5 & 35 & 4.8 & 0.93 & $0.49-1.75$ & 0.81 \\
\hline Writers, journalists & 8 & 2.6 & 21 & 2.9 & 0.88 & $0.39-2.01$ & 0.76 \\
\hline Artists & 2 & 0.6 & 3 & 0.4 & 1.59 & $0.26-9.58$ & 0.61 \\
\hline Photographers, photo processors & 7 & 2.2 & 12 & 1.7 & 1.38 & $0.54-3.54$ & 0.50 \\
\hline Printers & 5 & 1.6 & 13 & 1.8 & 0.91 & $0.32-1.39$ & 0.85 \\
\hline Salespersons & 102 & 32.6 & 247 & 34.0 & 0.94 & $0.71-1.25$ & 0.67 \\
\hline Clerks & 171 & 54.6 & 418 & 57.5 & 0.90 & $0.69-1.18$ & 0.45 \\
\hline Shippers & 9 & 2.9 & 32 & 4.4 & 0.65 & $0.31-1.38$ & 0.27 \\
\hline Messengers & 14 & 4.5 & 32 & 4.4 & 1.02 & $0.54-1.94$ & 0.95 \\
\hline Electronic equipment operators & 23 & 7.3 & 68 & 9.3 & 0.77 & $0.47-1.27$ & 0.31 \\
\hline Firemen & 0 & & 1 & 0.1 & $\cdot$ &.. & $\cdot$ \\
\hline Policemen, guards & 12 & 3.8 & 28 & 3.9 & 0.99 & $0.49-1.97$ & 0.97 \\
\hline Armed forces & 0 & & 2 & 0.3 & · & .. & · \\
\hline Janitors & 19 & 6.1 & 44 & 6.1 & 1.01 & $0.58-1.76$ & 0.98 \\
\hline Personal service workers & 37 & 11.8 & 95 & 13.1 & 0.90 & $0.60-1.35$ & 0.60 \\
\hline Textile workers & 3 & 1.0 & 12 & 1.7 & 0.59 & $0.16-2.11$ & 0.41 \\
\hline Food service workers & 97 & 31.0 & 204 & 28.1 & 1.15 & $0.86-1.54$ & 0.34 \\
\hline Food processors & 14 & 4.5 & 25 & 3.4 & 1.32 & $0.68-2.57$ & 0.42 \\
\hline Farm managers and workers & 16 & 5.1 & 35 & 4.8 & 1.07 & $0.58-1.96$ & 0.84 \\
\hline Fishermen, hunters, trappers & 0 & & 3 & 0.4 & $\cdot$ & .. & $\cdot$ \\
\hline Forestry and logging workers & 3 & 1.0 & 4 & 0.5 & 1.84 & $0.41-8.30$ & 0.43 \\
\hline Sawmill workers & 2 & 0.3 & 2 & 0.6 & 2.37 & $0.33-16.92$ & 0.39 \\
\hline Paper workers & 2 & 0.6 & 1 & 0.1 & 4.59 & $0.42-50.84$ & 0.21 \\
\hline Stone, glass and concrete workers & 0 & & 6 & 0.8 & $\cdot$ &.$\cdot$ & $\cdot$ \\
\hline Foundry and smelter workers & 0 & & 2 & 0.3 & $\cdot$ &.$\cdot$ & $\cdot$ \\
\hline Welders, cutters & 2 & 0.6 & 2 & 0.3 & 2.34 & $0.33-16.68$ & 0.40 \\
\hline Sheetmetal, iron and other metal workers & 3 & 1.0 & 7 & 1.0 & 1.03 & $0.26-4.02$ & 0.97 \\
\hline Chemical workers ${ }^{\mathrm{b}}$ & 2 & 0.6 & 4 & 0.5 & 1.17 & $0.21-6.41$ & 0.86 \\
\hline Construction workers & 3 & 1.0 & 6 & 0.8 & 1.18 & $0.29-4.75$ & 0.82 \\
\hline Electricians, electrical and electronics workers & 3 & 1.0 & 5 & 0.7 & 1.44 & $0.34-6.06$ & 0.62 \\
\hline Carpenters and woodworkers & 3 & 1.0 & 3 & 0.4 & 2.35 & $0.47-11.72$ & 0.30 \\
\hline Painters & 5 & 1.6 & 10 & 1.4 & 1.18 & $0.40-3.50$ & 0.76 \\
\hline Motor vehicle operators & 11 & 3.5 & 20 & 2.7 & 1.31 & $0.62-2.78$ & 0.48 \\
\hline Aircraft operators & 5 & 1.6 & 1 & 0.1 & 12.07 & $1.40-103.97$ & 0.02 \\
\hline Material moving equipment operators & 3 & 1.0 & 4 & 0.5 & 1.80 & $0.40-8.10$ & 0.44 \\
\hline Water transport workers & 0 & & 2 & 0.3 & $\cdot$ & .. & $\cdot$ \\
\hline Service station attendants & 4 & 1.3 & 2 & 0.3 & 4.81 & $0.87-26.41$ & 0.07 \\
\hline Vehicle mechanics & 1 & 0.3 & 4 & 0.5 & 0.60 & $0.07-5.36$ & 0.64 \\
\hline Mechanics $^{b}$ & 4 & 1.3 & 7 & 1.0 & 1.37 & $0.40-4.71$ & 0.62 \\
\hline Vehicle manufacturing & 0 & & 3 & 0.4 & $\cdot$ & .. & $\cdot$ \\
\hline Never worked outside the home & 8 & 2.6 & 17 & 2.3 & 0.99 & $0.40-2.43$ & 0.98 \\
\hline
\end{tabular}

${ }^{a}$ Category names are taken verbatim from the original paper. None of the following occupational categories were represented in the study group: metal miners, petroleum and gas workers, railway and other transport workers, boiler and transport firemen.

${ }^{\mathrm{b}}$ Not classified elsewhere. 
Table 3. Relationships between endometriosis case status and occupation, and case status and occupational duration, for selected occupations. (OR = odds ratio, $95 \% \mathrm{Cl}=95 \%$ confidence interval)

\begin{tabular}{|c|c|c|c|c|c|c|}
\hline \multirow{2}{*}{$\begin{array}{l}\text { Ever worked job and } \\
\text { duration worked }\end{array}$} & \multicolumn{2}{|c|}{ Cases } & \multicolumn{2}{|c|}{ Controls } & \multirow{2}{*}{\multicolumn{2}{|c|}{$\begin{array}{l}\text { Adjusted } \quad 95 \% \mathrm{Cl} \\
\text { OR }\end{array}$}} \\
\hline & N & $\%$ & N & $\%$ & & \\
\hline Biological scientists & 13 & 4.1 & 17 & 2.3 & $1.57^{b}$ & $0.73-3.36$ \\
\hline $\begin{array}{l}\text { For } \leq 5 \text { years } \\
\text { For }>5 \text { years }\end{array}$ & $\begin{array}{l}7 \\
6\end{array}$ & & $\begin{array}{r}13 \\
4\end{array}$ & . & $\begin{array}{l}1.24^{c} \\
3.15^{c}\end{array}$ & $\begin{array}{c}0.47-3.25 \\
0.85-11.70\end{array}$ \\
\hline Teachers, librarians & 53 & 16.9 & 158 & 21.7 & $0.74^{b}$ & $0.51-1.05$ \\
\hline $\begin{array}{l}\text { For } \leq 5 \text { years } \\
\text { For }>5 \text { years }\end{array}$ & $\begin{array}{l}30 \\
23\end{array}$ & & $\begin{array}{l}80 \\
78\end{array}$ & . & $\begin{array}{l}0.85^{c} \\
0.74^{c}\end{array}$ & $\begin{array}{l}0.53-1.35 \\
0.44-1.27\end{array}$ \\
\hline Health workers & 58 & 18.5 & 107 & 14.7 & $1.49^{b}$ & $1.03-2.15$ \\
\hline $\begin{array}{l}\text { For } \leq 5 \text { years } \\
\text { For }>5 \text { years }\end{array}$ & $\begin{array}{l}29 \\
29\end{array}$ & . & $\begin{array}{l}55 \\
52\end{array}$ & . & $\begin{array}{l}1.49^{c} \\
1.42^{c}\end{array}$ & $\begin{array}{l}0.92-2.43 \\
0.86-2.35\end{array}$ \\
\hline Wood/paper-related jobs ${ }^{d}$ & 9 & 2.9 & 10 & 1.4 & $2.16^{b}$ & $0.83-5.66$ \\
\hline Service station attendants ${ }^{\mathrm{e}}$ & 4 & 1.3 & 2 & 0.3 & $5.77^{\mathrm{b}}$ & $1.03-32.43$ \\
\hline Flight attendants ${ }^{f}$ & 5 & 1.6 & 1 & 0.1 & $9.80^{\mathrm{b}}-1$ & $1.08-89.0$ \\
\hline
\end{tabular}

a Reference category was those who had never worked this job.

${ }^{\mathrm{b}}$ Main category analyses are adjusted for age at reference date, reference year, alcohol use, oral contraceptive use, gravidity and nulliparity.

c Duration analyses are adjusted for age at reference date, reference year, education, tobacco use, oral contraceptive use, gravidity and nulliparity.

${ }^{d}$ No cases and 4 controls worked this job for more than 5 years.

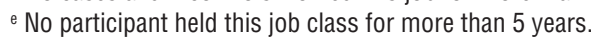

${ }^{\dagger}$ One case and no controls worked this job for 5 or fewer years.

Table 4. Relationships between endometriosis case status and occupation, and case status and occupational duration, for health workers. $(\mathrm{OR}=$ odds ratio, $95 \% \mathrm{Cl}=$ confidence interval)

\begin{tabular}{|c|c|c|c|c|c|c|}
\hline \multirow{2}{*}{$\begin{array}{l}\text { Ever worked job and } \\
\text { duration worked }\end{array}$} & \multicolumn{2}{|c|}{ Cases } & \multicolumn{2}{|c|}{ Controls } & \multirow{2}{*}{\multicolumn{2}{|c|}{$\begin{array}{l}\text { Adjusted } 95 \% \mathrm{Cl} \\
\text { OR }\end{array}$}} \\
\hline & N & $\%$ & N & $\%$ & & \\
\hline $\begin{array}{l}\text { Never worked a } \\
\text { health-related job }\end{array}$ & 255 & 81.5 & 620 & 85.3 & 1.00 & Reference \\
\hline Physicians ${ }^{\text {a }}$ & 2 & 0.6 & 1 & 0.1 & $7.80^{\circ}$ & $0.62-97.83$ \\
\hline $\begin{array}{l}\text { Registered and licensed } \\
\text { practical nurses }\end{array}$ & 20 & 7.3 & 28 & 4.3 & $1.72^{b}$ & $0.93-3.19$ \\
\hline $\begin{array}{l}\text { For } \leq 5 \text { years } \\
\text { For }>5 \text { years }\end{array}$ & $\begin{array}{r}6 \\
14\end{array}$ & $\cdot$ & $\begin{array}{r}6 \\
22\end{array}$ & $\cdot$ & $\begin{array}{l}3.23^{\mathrm{c}} \mathrm{C} \\
1.45^{\mathrm{c}}\end{array}$ & $\begin{array}{c}0.95-11.06 \\
0.71-2.98\end{array}$ \\
\hline Nursing and health aides & 35 & 12.1 & 64 & 9.4 & $1.57^{b}$ & $0.99-2.47$ \\
\hline $\begin{array}{l}\text { For } \leq 5 \text { years } \\
\text { For }>5 \text { years }\end{array}$ & $\begin{array}{l}26 \\
11\end{array}$ & $\dot{.}$ & $\begin{array}{r}53 \\
9\end{array}$ & $\dot{.}$ & $\begin{array}{l}1.42^{c} \\
2.18^{c}\end{array}$ & $\begin{array}{l}0.85-2.36 \\
0.85-5.54\end{array}$ \\
\hline Radiologic technicians ${ }^{d}$ & 1 & 0.4 & 3 & 0.5 & $0.81^{b}$ & $0.08-8.05$ \\
\hline Other health workers ${ }^{d}$ & 14 & 5.2 & 28 & 4.3 & $1.31^{b}$ & $0.66-2.62$ \\
\hline
\end{tabular}

a No participant held this job class for $<5$ years.

${ }^{b}$ Main category analyses are adjusted for age at reference date, reference year, alcohol use, oral contraceptive use, gravidity and nulliparity.

c Duration analyses are adjusted for age at reference date, reference year, education, tobacco use, oral contraceptive use and gravidity.

${ }^{\mathrm{d}}$ No participant held this job class for more than 5 years.
Table 5. Relationships between occupation and manifestation of endometriosis for selected occupations. (OR = odds ratio, $95 \% \mathrm{Cl}$ $=95 \%$ confidence interval)

\begin{tabular}{lrrr}
\hline \multirow{2}{*}{ Occupation } & \multicolumn{3}{c}{ Non-infertile cases only (N=260) } \\
\cline { 2 - 4 } & $\mathrm{N}$ & $\begin{array}{c}\text { Adjusted } \\
\text { OR }\end{array}$ & \multicolumn{1}{c}{$95 \% \mathrm{Cl}$} \\
\hline Biological scientists $^{\mathrm{b}}$ & 13 & 1.78 & $0.82-3.85$ \\
Teachers, librarians $^{\mathrm{b}}$ & 53 & 0.86 & $0.60-1.13$ \\
Health workers $^{\mathrm{b}}$ & 56 & 1.41 & $1.06-2.22$ \\
Physicianss $^{\mathrm{c}}$ & 2 & 9.05 & $0.71-115.34$ \\
Registered nurse or $_{\text {licensed practical nurse }^{\mathrm{c}}}$ & 20 & 1.80 & $0.97-3.34$ \\
Nursing, health aides $^{\mathrm{c}}$ & 33 & 1.60 & $1.00-2.55$ \\
Radiologic technologists $^{\mathrm{c}}$ & 1 & 0.82 & $0.08-8.11$ \\
Other health workers $^{\mathrm{c}}$ & 14 & 1.38 & $0.70-2.76$ \\
Wood/paper-related jobs $^{\mathrm{b}}$ & 9 & 2.29 & $0.88-5.99$ \\
Service station attendants $^{\mathrm{b}}$ & 4 & 5.98 & $1.07-33.45$ \\
Flight attendants $^{\mathrm{b}}$ & 5 & 10.18 & $1.12-92.14$ \\
\hline
\end{tabular}

a Adjusted for age at reference date, reference year, alcohol use, oral contraceptive use, gravidity, and nulliparity.

${ }^{b}$ Reference category was those who had never worked this job.

c Reference category was those who had never worked a health-related job.

at increased endometriosis risk, (respectively, OR 1.49, 95\% CI 1.03-2.15; OR 5.77, 95\% CI 1.03-32.43; and OR 9.80, 95\% CI 1.08-89.02). Teachers/librarians were at non-significantly decreased endometriosis risk (OR 0.74, 95\% CI 0.51-1.05), while working in a wood/paper-related job or as a biological scientist was associated with a non-significantly increased risk of disease (respectively, OR 2.16, 95\% CI 0.83-5.66; and OR 1.57, 95\% CI 0.73-3.36). Working in an occupation for five years or longer did not appear to strengthen negative or positive associations with that occupation.

In analyses of subcategories of health care workers (table 4), non-significant elevations in disease risk were seen among physicians, registered nurses/licensed practical nurses, and nursing aides/health aides (respectively, OR 7.80, 95\% CI 0.62-97.83; OR 1.72, 95\% CI 0.93-3.19; and OR 1.57, 95\% CI 0.99-2.47).

The associations between selected occupations and case status by presentation are shown in table 5 . In general, associations between occupation and case status were more pronounced among those presenting for reasons other than infertility than among all cases. Those whose primary disease manifestation was not infertility were more likely to have worked as service station attendants (OR 5.98, 95\% CI 1.07-33.45), flight attendants (OR 10.18, 95\% CI 1.12-92.14), or health workers (OR 1.53, 95\% CI 1.06-2.22), including registered nurses/licensed practical nurses (OR 1.80, 95\% CI 0.97-3.34), and nursing/health aides (OR 1.60, 95\% CI 1.00-2.55). 


\section{Discussion}

Our exploratory study suggested that having ever worked as a flight attendant, service station attendant, or health worker, particularly as a nurse, may be associated with increased endometriosis risk. There was some indication that having worked as a teacher or librarian is associated with a decreased risk, although the association was not statistically significant. Some potential exposures that were found among one or more of the vulnerable jobs, but uncommon in school or library settings, included (i) radiation (health workers and flight attendants), (ii) fuel and exhaust components (service station and flight attendants), (iii) night work (all), and (iv) circadian/sleep disruption (all). Another study of this population suggested that endometriosis may be related to night and evening shift work (11), but small numbers prevented an examination of risk by job title in that analysis.

Measuring exposures is preferable to using job titles in occupational health research (16). However, since little is known about occupation and endometriosis, and the present study did not allow the measurement of a spectrum of exposures, using job categories provided an alternative exploratory approach to generate initial occupational hypotheses. Occupations include a broad array of exposures and may also capture some aspects of socioeconomic standing. In this study, income and education did not affect the OR estimates for the occupations examined, suggesting that physical exposures are more likely than sociodemography to be responsible for any associations seen. We also found that an occupational duration of $\geq 5$ years did not appear to strengthen the associations within the occupational classes studied. The absence of a duration dose response might suggest that a shared chemical exposure is not responsible for the association, but larger studies are required to address this hypothesis more definitively. We did not find that women with endometriosis were less likely to work than women without the disease, but if women with severe symptoms were less likely to belong to the health-maintenance organization under study (perhaps they were not working at all), associations between disease risk and occupations would be attenuated.

The most commonly studied compounds likely to be occupationally related to endometriosis are dioxins and other organochlorine compounds. Associations between endometriosis and these substances have been controversial, with most studies involving simultaneous measurement of body burden and disease status (17-19), or measurements after industrial disasters (20), rather than routine occupational exposures. Previous occupational studies of infertility in relation to certain industries and occupations have evaluated endometriosis as a secondary outcome. Taskinen et al found substantially elevated endometriosis risks among woodworkers exposed to formaldehyde and organic solvents (formaldehyde OR 4.5, 95\% CI 1.0-20.0; organic solvents OR 14.7, 95\% CI 3.1-70.0), but the presence of endometriosis was ascertained from self-report in that study, and temporal relationships between exposure and outcome could not be determined (7). The suggestive but non-significant association we found between endometriosis and wood/paper-related jobs may support these findings.

In contrast to our results, Fuortes et al found strong associations between endometriosis and working in agricultural industries (OR 6.9, 95\% CI 1.6-30.4) and occupations (OR 8.1, 95\% CI 1.3-52.5) when comparing women with medically confirmed infertility caused by endometriosis to postpartum women (9). The present work compared women with surgically confirmed endometriosis (with and without infertility) to a general population of female members of a health-maintenance organization. The different control populations affect the interpretation of the $\mathrm{OR}$ - one reflects the relative risk of exposure among those who are infertile due to endometriosis versus those who are fertile, while the other reflects exposure among those with and without endometriosis irrespective of fertility. One possible explanation of the difference in findings is that endometriosis might be equally likely to occur among women who did or did not work in agricultural occupations, but more likely to be diagnosed as the cause of infertility, or to cause infertility, among those who had agricultural occupations.

This was the first systematic survey of an occupational risk of endometriosis. This study had a number of important strengths. The study design allowed nearly complete case ascertainment. Instead of the potentially biased samples obtained by recruiting patients from gynecology or infertility clinics, this design enabled the full spectrum of disease diagnosed within a large health-maintenance organization to be represented. For this analysis, we excluded cases without surgical confirmation, in contrast to previous occupational studies that have relied on self-report. We also excluded all those who reported prior surgically confirmed endometriosis to capture the first diagnosis and look at exposures in the correct timeframe. Experts evaluated disease features and its course directly from medical records rather than relying on self-report, and we used a well-defined set of criteria to evaluate the certainty of diagnosis, enhancing the reproducibility of our findings. We did rely, however, on self-reported occupational histories. If inaccurate, these reports could produce a biased risk estimate, particularly if cases and controls recalled occupations differently, but such histories have been found to have high validity and reliability (21), and the interview covered many topics, so occupation was 
not likely to be seen as a special exposure under study and recalled in more detail by either group.

As well, investigators seeking to evaluate the effects of occupational exposures on reproductive health may wish to take into consideration the infertile worker effect, whereby women raising families are likely to spend less time in the workforce than their counterparts so that paid employment may be falsely associated with reproductive disease risk (22). To address this issue, we adjusted risk estimates for gravidity and nulliparity, and evaluated non-infertile cases separately. These adjustments did not substantively affect the general relationships between being employed in a particular job class and endometriosis. The infertile worker effect may have a more substantial impact on the relationship between reproductive disease and overall employment or duration of employment, rather than employment in a particular type of job.

In this study, we used the 1980 Census Occupational Codes. While the codes are dated and somewhat arbitrarily numerically arranged, we chose to use them because they formed the basis for the Schnitzer et al aggregation scheme, which overcomes the irregularities of grouping by, for instance, classifying all health care professionals together. However, this cannot overcome the limitation of aggregating disparate occupations into single codes, such as the inclusion of registered nurses, nurses in advanced practice occupations requiring graduate and postgraduate education (nurse midwife and nurse anesthetist), and the exclusion of administrative nursing positions in code 095 . A second shortcoming was the absence of codes for new but well-populated professions, such as web designer, network engineer, and help desk, and industries like information technology and biotechnology. These codes, however, are broadly used in studies of population-level occupational data (23) and the Schnitzer et al categorization was explicitly developed for use with this version of the codes in studies of workplace exposures.

This exploratory study suggested that there may be an associated increased risk of endometriosis for those women who have ever worked as a flight attendant, service station attendant, or health worker, particularly as a nurse. An examination of endometriosis risk in existing occupational cohorts may be a beneficial next step to better understand the role of occupational exposures in the etiology of endometriosis.

\section{Acknowledgements}

This study received support from: (i) the National Institutes of Health (NIH) grant numbers R01 HD33792,
F31 NR009164, P30 CA015704, and T32 HD052462; (ii) the maternal and child health dissertation award from the Maternal and Child Health Leadership Training Program at the University of Washington School of Public Health and Community Medicine; and (iii) the Woodrow Wilson-Johnson \& Johnson dissertation grant in women's health.

Thanks to the participants of the study; Dean Nancy Fugate Woods for her comments on the manuscript, Berta Nicol-Blades and Dr. Anneclaire De Roos for their technical assistance, and Georgia Green for her administrative support.

\section{References}

1. Holt VL, Jenkins J. Endometriosis. In: Goldman M, Hutch M, editors. Women and health. San Diego (CA): Academic Press; 2000. p 226-39.

2. Brinton LA, Gridley G, Persson I, Baron J, Bergqvist A. Cancer risk after a hospital discharge diagnosis of endometriosis. Am J Obstet Gynecol. 1997;176(3):572-9.

3. Lee KR, Nucci MR. Ovarian mucinous and mixed epithelial carcinomas of mullerian (endocervical-like) type: a clinicopathologic analysis of four cases of an uncommon variant associated with endometriosis. Int J Gynecol Pathol. 2003;22(1):42-51.

4. Swiersz LM. Role of endometriosis in cancer and tumor development. Ann N Y Acad Sci. 2002;955:281-5.

5. Swiersz LM. Role of endometriosis in cancer and tumor development. Ann N Y Acad Sci. 2002;955:396-406.

6. Modesitt SC, Tortolero-Luna G, Robinson JB, Gershenson DM, Wolf JK. Ovarian and extraovarian endometriosis-associated cancer. Obstet Gynecol. 2002;100(4):788-95.

7. Taskinen HK, Kyyrönen P, Sallmén M, Virtanen SV, Liukkonen TA, Huida $\mathrm{O}$, et al. Reduced fertility among female wood worker exposed to formaldehyde. Am J Ind Med. 1999;36:206-12.

8. Smith EM, Hammonds-Ehlers M, Clark MK, Kirchner HL, Fuortes L. Occupational exposures and risk of female infertility. J Occup Environ Med. 1997;39(2):138-47.

9. Fuortes L, Clark AJ, Kirchner HL, Smith EM. Association between female infertility and agricultural work history. Am J Ind Med. 1997;31:445-51.

10. US Department of Labor Bureau of Labor Statistics. Women in the labor force: a databook. Washington (DC): US Government Print Office; 2005.

11. Marino JL, Holt VL, Chen C, Davis S. Shift work, hCLOCK T3111C polymorphism, and endometriosis risk. Epidemiology. 2008;19(3):477-84.

12. Holt VL, Weiss NS. Recommendations for the design of epidemiologic studies of endometriosis. Epidemiology. 2000;11(6):654-9. 
13. US Department of Commerce. 1980 census of population: alphabetical index of industries and occupations. Washington (DC): US Government Printing Office; 1982.

14. US Department of Commerce. Standard occupational classification manual. Washington (DC): US Government Printing Office; 1980.

15. Schnitzer PG, Teschke K, Olshan AF. A classification scheme for aggregating U.S. Census Occupation and Industry codes. Am J Ind Med. 1995;28:185-91.

16. Messing K, Punnet L, Bond M, Alexanderson K, Pyle J, Zahm S, et al. Be the fairest of them all: challenges and recommendations for the treatment of gender in occupational health research. Am J Ind Med. 2003;43:618-29.

17. Pauwels A, Schepens PJC, D'Hooghe T, Delbeke L, Dhont M, Brouwer A, et al. The risk of endometriosis and exposure to dioxins and polychlorinated biphenyls: a case-control study of infertile women. Hum Reprod. 2001;16(10):2050-5.

18. Heilier J-F, Nackers F, Verougstraete V, Tonglet R, Lison D, Donnez J. Increased dioxin-like compounds in the serum of women with peritoneal endometriosis and deep endometriotic (adenomyotic) nodules. Fertil Steril. 2005;84(2):305-12.

19. Tsukino H, Hanaoka T, Sasaki H, Motoyama H, Hiroshima M, Tanaka T, et al. Associations between serum levels of selected organochlorine compounds and endometriosis in infertile Japanese women. Environ Res. 2005;99(1):118-25.

20. Eskenazi B, Mocarelli P, Warner M, Samuels S, Vercellini P, Olive DL, et al. Serum dioxin concentrations and endometriosis: a cohort study in Seveso, Italy. Environ Health Perspect. 2002;110(7):629-32.

21. Teschke K, Olshan AF, Daniels JL, De Roos AJ, Parks CG, Schulz M, et al. Occupational exposure assessment in casecontrol studies: opportunities for improvement. Occup Environ Med. 2002;59:575-94.

22. Joffe M. Biases in research on reproduction and women's work. Int J Epidemiol. 1985;14(1):118-23.

23. Park RM, Schulte PA, Bowman JD, Walker JT, Bondy SC, Yost MG, et al. Potential occupational risks for neurodegenerative disease. Am J Ind Med. 2005;48:63-77.

Received for publication: 12 August 2008 